\title{
Hormonal profile and characteristics of polychistic ovarium syndrome among Madurese tribe, Indonesia
}

\author{
Zakkiyatus Zainiyah, Eny Susanti, Mustofa Haris \\ Ngudia Husada Madura College of Health Sciences (STIKes), Indonesia
}

\section{Article Info}

Article history:

Received Jan 23, 2020

Revised Aug 10, 2020

Accepted Sep 5, 2020

\section{Keywords:}

Body mass index (BMI)

Follicle-stimulating hormone Luteinizing hormone (LH) Polycystic ovarium syndrome Weight

\begin{abstract}
Polycystic ovarium syndrome (PCOS), the most common endocrine disorders in women of reproductive age, characterized by menstrual disorders (amenorrhea/oligomenorrhea), hirsutism, the appearance of acne, alopecia and the results of biochemical tests that show increased androgens (testosterone). Increased serum luteinizing hormone (LH) and follicle stimulating hormone (FSH) serve as diagnostic tests for PCOS over the years, but from several research results obtained inconsistent results that need further research. The purpose of this study is to analyze the hormonal profile and characteristics of polycystic ovary syndrome (PCOS) in Madura. Case-control study conducted in March-August 2019 in the Madura tribe. There were 32 subjects with PCOS and 32 healthy women participated in this study. Hormonal examination was using a serum and followed by enzymelinked immune sorbent assay (ELISA kit). Levels Follicle-stimulating hormone (FSH) average was 8.74 and SHBGs were 10.02 lower in PCOS patients and LH levels were higher in PCOS patients; ratio LH/FSH was 0.76. The results of the Madurese study showed that levels of FSH, LH, weight significantly related to PCOS sex hormone binding globulin (SHBG), however body mass index (BMI) levels were not related to PCOS.
\end{abstract}

This is an open access article under the CC BY-SA license.

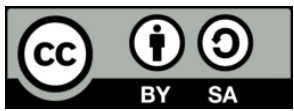

\section{Corresponding Author:}

Zakkiyatus Zainiyah,

Department of Professional Midwifery,

Ngudia Husada Madura Institute of Health Sciences (STIKes),

Jl. RE. Martadinata No 45, Mlajah Bangkalan, Indonesia.

Email: zzainiyah@gmail.com

\section{INTRODUCTION}

Polycystic ovary syndrome (PCOS) is one of the most common endocrine disorders in women of reproductive age, characterized by menstrual disorders (amenorrhea/oligomenorrhea), hirsutism, emergence of polycystic ovary syndrome (PCOS), one of the most common endocrine disorders in women of reproductive age, characterized by menstrual disorders (amenorrhea/oligomenorrhea), hirsutism, emergence of polycystic ovary syndrome (PCOS) the results of biochemical examination showed that androgens (testosterone) were increased [1-3]. Long-term effects of PCOS usually occur with infertility, insulin resistance, increased risk of diabetes and cardiovascular disease [4, 5]. El Hayek said the prevalence of PCOS is around 4\% to $10 \%$ of women worldwide [1].

There are three criteria for the diagnosis of PCOS, namely the national institutes of health $(\mathrm{NIH})$ Criteria, the criteria for androgen excess and polycystic ovarian syndrome society (AE-PCOS), and Rotterdam criteria. The Rotterdam Criteria is a consensus that resulted from a conference organized by the European society for human reproduction and embryology/the American society for reproductive medicine (ESHRE/ASRM) in 2003. A diagnosis of PCOS is established by the Rotterdam criteria if two of the following 
three criteria are found, namely dysfunction ovaries, hyperandrogenic manifestations, and polycystic ovary morphological features. The Rotterdam Criteria are the criteria used for handling infertility $[6,7]$.

In PCOS patients there is an increase in the hormone's LH and FSH as much as 70\% or 2-3 times that of normal women. Increased serum LH and FSH serve as diagnostic tests for PCOS over the years, but from several research results obtained inconsistent results. Several studies reported serum levels LH basal inversely correlated with body mass index (BMI) in PCOS patients, who did not find an association between BMI and levels LH in PCOS patients [8]. An increase in LH occurs in thin and normal women whereas a decrease in LH is even normal in obese women [9]. So that early detection of PCOS is needed more consistently and earlier because early detection of PCOS determines the success of therapy.

Reproductive hormonal disorders are often found in PCOS. Increased function of luteinizing hormone $(\mathrm{LH})$ is an important factor in the pathophysiology of PCOS that causes ovarian theca cells to produce excess androgen hormones so that there is a state of hyperandrogenism. The state of hyperandrogenism causes a disturbance in the development of ovarian follicles so that the follicular maturation process is inhibited. This condition causes the follicle growth to stop at a diameter of $2-9 \mathrm{~mm}$ and produces a polycystic ovary appearance [10]. Research on examining hormonal levels and characteristics in PCOS patients has never been done based on ethnicity. This study is to analyze the profile of hormonal levels and characteristics of Polycystic Ovary Syndrome in Madura.

\section{RESEARCH METHOD}

This study used case-control, where there are two groups, PCOS cases and healthy women control with a total sample of 64. The criteria of a control group of native Madurese women, aged 20-40 years and already married, currently were not using hormonal contraception three months before, did not have diseases such as sugar, liver and kidney levels, while the case group, native Madurese women, aged 20-40 years and married, did not have diabetes, liver and kidney disease, women with first pregnancy program. Sampling was carried out during the 3-5 days of menstruation and morning. Every patient besides blood drawn weight and height examination to measure BMI and continued blood drawn to take serum followed by an examination their levels of the hormone FSH, LH and SHBG on all respondents by using the ELISA kit. FSH uses ELISA kit 180670, sex hormone binding globulin (SHBG), enzyme-linked immune sorbent assay (ELISA) kit 96T, and LH ELISA kit 4040. The examination material is derived from venous blood of research subjects obtained through a phlebotomy procedure, and then the examination material is left for 30 minutes until a clot is formed. The test material is centrifuged at a speed of $1,000 \mathrm{~g}$ for 20 minutes until the serum is formed. Afterward, serum is stored at $-800 \mathrm{C}$ until the required sample size met 64 respondents. Previously all respondents were screened for serum glutamic oxaloacetic transaminase (SGOT), blood urea nitrogen (BUN), creatinine and blood sugar to ensure that the respondents were in good conditions and will continued for further examination. But if there were any respondents did not meet the criteria then they will be excluded.

The study protocol was approved by Ngudia Husada Madura Institute of Health (STIKes) and Eligible Ethics No. 0034/KEPK-NHM/EC/II/2019. To find out the differences between the case and control groups both respondent characteristics and hormonal levels we were using the Mann-Withney statistical test. To find out which variables have significant effect on PCOS, we were using logistic regression with a significance level of 0.05. Statistical analysis was using the Statistical Package for the Social Sciences (SPSS) version 21.0 for windows.

\section{RESULTS AND DISCUSSION}

\subsection{Distribution of clinical symptoms in study subjects}

Table 1 shows distribution of clinical symptoms among respondent of secondary amenorrhea, hirsutism, acne great sound, alopecia. Based on Table 1 it is found that most of the study subjects in PCOS patients have 2-3 clinical symptoms, and most often clinical symptoms that appear are amenorrhoea, hirsutism and the appearance of acne.

Table 1. Distribution of clinical symptoms in study subjects

\begin{tabular}{cc}
\hline Variable & $\mathrm{n}=\%$ \\
\hline Secondary Amenorrhea & $30(100)$ \\
Hirsutism & $19(63.33)$ \\
Acne & $18(60)$ \\
Great sound & $2(6.7)$ \\
Alopecia & $0(0)$ \\
\hline
\end{tabular}

Int. J. Public Health Sci, Vol. 9, No. 4, December 2020: 333 - 337 
Table 2 shows result of respondent age, weight, height, BMI and LH/FSH. Based on Table 2, it was found that the average age of PCOS was 30.57 years and control was 25.27 years, BMI of research subjects was an average of 26.06 and controls 22.55 then weight research subjects were average mean 63.56 and control 53.07. There was a difference in weight, BMI ratio FSH and LH with $\mathrm{p}<0.05$, and there was no difference for height because $p<0.05$. Due to the data were not normally distributed $p<0.05$ then was using the statistical test Mann-Withney. Table 3 shows the logistic regression test.

Table 2. Result of research subjects

\begin{tabular}{cccc}
\hline Characteristics of & PCOS & Control & p-value \\
\hline Age & $30.57 \pm 5.37$ & $25.27 \pm 4.93$ & 0.000 \\
Weight & $63.56 \pm 13.97$ & $53.07 \pm 5.99$ & 0.003 \\
Height & $155.7 \pm 6.47$ & $154.3 \pm 5.04$ & 0.571 \\
BMI & $26.06 \pm 4.63$ & $22.55 \pm 2.16$ & 0.003 \\
LH/FSH & $0.76 \pm 0.50$ & $0.43 \pm 0.20$ & 0.009 \\
Value of normality Shapiro Wilk & & & 0.041 \\
\hline
\end{tabular}

Table 3. Logistic regression test result

\begin{tabular}{cccccc}
\hline Variabel & $\beta$ & Signifikan & $\operatorname{Exp}(ß)$ & Lower & Upper \\
\hline Age & 0.235 & $0.002^{*}$ & 1.265 & 1.089 & 1.471 \\
Weight & 0.095 & $0.022^{*}$ & 1.100 & 1.014 & 1.193 \\
Height & -0.235 & 0.752 & 0.791 & 0.184 & 3.397 \\
BMI & -0.304 & 0.343 & 0.738 & 0.394 & 1.383 \\
FSH (IU/I) & -0.254 & $0.014^{*}$ & 0.776 & 0.634 & 0.950 \\
LH (IU/I) & 0.384 & $0.018^{*}$ & 1.468 & 1.069 & 2.015 \\
SHBG (IU/I) & -0.002 & 0.259 & 0.998 & 0.994 & 1.002 \\
\hline
\end{tabular}

Results of the current research show that age in the PCOS group were on average 30 years old and in the control group 25 years old, seen from the age in the range of healthy reproductive age. PCOS also often occurred in adolescents, this is in accordance with research conducted by Fazleen, that in adolescents there were many metabolic syndromes associated with PCOS. However, several studies explained that many PCOS identified/diagnosed at reproductive age [11]. At the reproductive age, the quality of eggs should be in good condition, but because of obesity and imbalance of the hormones FSH and LH, it will affect the quality of the eggs to shrink, which will result in PCOS.

Based on research conducted by Pinola revealed that at age $>39$ years, BMI and waist circumference were higher in the hyper androgenic PCOS group than in the normal androgen PCOS group [12]. This is in line with research conducted in the Madura tribe that the PCOS group had a BMI in the obesity category at the age of $>30$ years. The results of this study also showed in the PCOS group, BMI were in the $50 \%$ obesity category and $50 \%$ normal, while in the BMI control group, there were in the normal categories, signs, and symptoms of PCOS one of which is irregular menstruation due to obesity, this is in accordance with the Mahedia 2018 study, said that women who are obese will increase the occurrence of irregular menstruation causing infertility [13, 14].

This parallel study conducted by Jamil, revealed that BMI was significant in the Aotipers (Oligo anovulation, hyper androgenic and PCOS) [14]. Research conducted in Norway, 2001 that BMI did not correlate with insulin resistance, but did affect the decrease in egg cell quality and increase in FSH [15]. The study also revealed that $50 \%$ of PCOS were not obese [16]. Another study conducted by Diamanti, revealed that $40-60 \%$ of PCOS women are overweight or obese, and this is widely understood and accepted then obesity is also associated with health risks $[17,18]$.

The results of this study were based on the characteristics of PCOS patients and healthy women found, weight, BMI had a significant relationship, weight and BMI in PCOS patients were higher than in the control group, and this was consistent with Bassiuny, research that BMI was significantly higher in the PCOS group [19]. This study is also in line with Mukherjee, 2009 said that BMI and weight were significantly higher than controls [20]. A study conducted by Martinez, 2007 said obesity and overweight are clinical manifestations of PCOS, especially obesity that occurs in the abdominal area; even $50 \%$ of women who are obese or overweight will develop PCOS [21].

Based on those research reports, it can be seen that BMI was a risk factor for PCOS [22]. Excess deposits in the abdomen, especially in the visceral part are indicative of an increase in androgens in the ovary that triggers PCOS [23]. Moran and Teede, reported that abdominal adipocytes are a risk factor for metabolic disorders in PCOS patients. Hyperandrogenism and insulin resistance can affect metabolic abnormalities either directly or indirectly in women who are obese especially in the abdominal region [24]. Research conducted on Indian women with a sample of 63 obese PCOS patients revealed that the characteristics of anthropometry especially BMI and pelvic circumference were the most important parameters and were 
highly related to the incidence of PCOS compared to insulin resistance in obese PCOS patients, so it was very important for treatment premature obesity to prevent dyslipidemia in the future [25]. It was reported that $\mathrm{LH}$ levels, ratio LH/FSH was higher in the PCOS group than in the normal group, whereas SHBG levels found to be lower in the PCOS group than in the normal group [22]. When puberty occurs physiologically there is an increase of insulin in the body there will be a decrease SHBG levels which will increase androgen levels in the ovary by stimulating the ovaries directly, in women with PCOS, physiologically hyperinsulinemia has occurred in adolescence and triggers hyper androgenic and anovulation, teenagers who tend to insulin resistance and being overweight are risk factors for PCOS [26].

This study is in line with research in the Madura tribe which found higher LH levels and lower SHBG levels in the PCOS group compared to the control group. While the levels of FSH, LH, and SHBG there was no difference between the PCOS group and the normal group. And the results of the ratio LH/FSH found there were significant differences between cases and controls in the Madura tribe. The current diagnosis of PCOS can be seen from the results of the LH/FSH ratio, if the ratio LH/FSH $>2$ then the possibility of a diagnosis of PCOS is greater. Judging from the relationship test, FSH levels and LH there is a significant relationship between PCOS. In women of reproductive age, FSH is an important hormone needed for follicular development, oocyte maturation, and regulation of steroidogenesis in the ovaries. Research conducted on adolescents in Turkey experiencing PCOS found that BMI, waist circumference, LH levels and ratios LH/FSH was higher in the PCOS than in the control group, whereas FSH and SHBG levels were lower in PCOS than in the PCOP group control [23]. The same study was also carried out in Turkey in adolescents who experience hirsutism in PCOS, reporting that the ratio LH/FSH is higher and SHBG levels are lower and really significant in the incidence of PCOS [27].

One of the common clinical manifestations in PCOS patients is an assessment of the difference in the range between LH and FSH levels through the ratio LH/FSH when >2 is said to be PCOS [28]. In women with PCOS, an increase in LH levels will secrete excess androgens in the ovaries, and FSH levels can stimulate cells in the ovary to convert excess androgens into estrogen. In certain women, excess levels LH can not only cause high androgen levels from theca and ovarian cells but can also increase estrogen from other cells in the ovary [29]. Previous research reported that there was no significant difference between the obese and non-obese groups in PCOS patients and from the results of FSH, LH levels and also ratio of LH/FSH [30]. This is in line with this study that there was no significant difference between the case and control groups, but from the logistic regression results, there is a significant relationship between LH and FSH with the incidence of PCOS.

A high percentage (55-75\%) of women with PCOS have high ratios of LH/FSH probably due to higher levels of LH than decreased production of FSH. Stimulation GnRH causes the production of LH excessive in women. This condition can be determined by the highest frequency or amplitude of GnRH. On the other hand, PCOS patients have higher and lower concentrations LH and FSH than normal women [26]. Research conducted by Jamil revealed that women with D-suppressors (Oligo anovulation and PCOS) showed LH/FSH significantly lower ratios, testosterone levels, and androgen levels. FSH and SHBG levels were higher than those with an Oligo anovulation, hyperandrogenic and oscillator PCOS [14]. This study is not in line with research conducted in Madura that found lower FSH and SHBG levels as well as higher LH levels in PCOS group. Research in Madura was in line with Turhan's 1999 studied of 32 PCOS patients and controls of 25 healthy women revealed that LH levels were higher and SHBG and FSH levels were lower in PCOS [30]. The path analysis found that hormone level is very significantly related to PCOS are FSH levels and LH levels. This is in line with research conducted by Turhan revealed that the combination of both hormone FSH levels and LH can be used to predict of PCOS, especially in the ratio of FSH/LH [30].

\section{CONCLUSION}

Most of the Madurese tribe in Indonesia, age and weight contributed to BMI. Waist circumference was higher in the hyper androgenic PCOS group than in the normal androgen PCOS group. FSH levels and LH levels were significantly related PCOS. The combination of both hormone FSH levels and LH can be used to predict of PCOS, especially in the ratio of FSH/LH. Among reproductive age women, FSH is an important hormone needed for follicular development, oocyte maturation, and regulation of steroidogenesis in the ovaries.

\section{REFERENCES}

[1] El Hayek S., Bitar L., Hamdar LH., Mirza FG., Daoud G., "Poly Cystic Ovarian Syndrome: An updated overview," Front Physiol., vol. 7, no. 124, pp. 1-15, 2016.

[2] Manlove HA. Polycystic ovary syndrome (PCOS) in urban India. UNLV Theses, Dissertations, Professional Papers, and Capstones. 936., May 2011. [Online]. Available: https://digitalscholarship.unlv.edu/thesesdissertations/936. 
[3] Ding T., Baio G., Hardiman PJ., Petersen I., Sammon C., "Diagnosis and management of polycystic ovary syndrome in the UK): a retrospective cohort study (2004-2014)," BMJ open, vol. 6, no. 7, pp. 1-8. 2016.

[4] Thomson RL, Buckley JD, Brinkworth GD. Perceived exercise barriers are reduced and benefits are improved with lifestyle modification in overweight and obese women with polycystic ovary syndrome: a randomized controlled trial. BMC Womens Health., vol. 16, no. 14, pp. 1-8, 2016.

[5] Musmar S, Afaneh A, Mo'alla H., "Epidemiology of polycystic ovary syndrome: a cross sectional study of university students at An -Facial national university-Palestine," Reprod Biol Endocrinol., vol. 11, no .1, pp. 47-52. 2013.

[6] Gourgari E, Spanakis E, US Dobs. Pathophysiology, risk factors, and screening methods for prediabetes in women with polycystic ovary syndrome. International Journal Womens Health, vol. 8, no. 2, pp. 381-7, 2016.

[7] BCJM Fauser., "Revised 2003 consensus on diagnostic criteria and long-term health risks related to polycystic ovary syndrome," Sterile Fertil., vol. 81, no, 1, pp.19-25, 2004.

[8] Ramezanali F., Khalili G., Arabipoor A., Bagheri Lankarani N., Moini A., "Relationships between Serum Luteinizing Hormone Level, Endometrial Thickness and Body Mass Index in Polycystic Ovary Syndrome Patients with and without Endometrial Hyperplasia," Int J Fertil Sterile., vol. 10, no. 1, pp. 36-41. 2016.

[9] Benson RC., "Clinical Gynecologic Endocrinology and Infertility,” West J Med., vol. 121, no. 2. pp. 52-64, 1974.

[10] Mustari DA, Rostini T, Indrati AR, Bayuaji H RS., "Correlation of Amtral Follicular Amounts with Serum 25 (OH) D Levels in Patients with Polycystic Ovary Syndrome Correlation between Antral Follicles Count and Serum 25 (OH) D Levels in Polycystic Ovary Syndrome Patients 2018," Majalah Kedokteran Bandung, vol. 50, no 4, pp. 4-9. 2018.

[11] Fazleen NE., Whittaker M., Mamun A., "Risk of metabolic syndrome in adolescents with polycystic ovarian syndrome: A systematic review and meta-analysis," Diabetes Metab Syndr Clin Res Rev., vol. 2, no. 4, pp. 1-8, 2018.

[12] Pinola P, Puukka K, Piltonen TT, Puurunen J, Vanky E, Sundström-Poromaa I, et al., "Normo-and hyperandrogenic women with polycystic ovary syndrome exhibit an adverse metabolic profile through life," Sterile Fertil., vol. 107, no. 3, 788-795. 2017.

[13] Maredia H., Hawley NL., Lambert-Messerlian G., Fidow U., Reupena MS., Naseri T., et al., "Reproductive health, obesity, and cardiometabolic risk factors among Samoan women," Am J Hum Biol., vol. 3, no. 6, pp. 45-53, 2018.

[14] Jamil AS, Alalaf SK, Al-Tawil NG, Al-Shawaf T., "Comparison of clinical and hormonal characteristics among the four phenotypes of polycystic ovary syndrome based on the Rotterdam criteria," Arch Gynecol Obstet., vol. 293, no. 2, pp. 447-56, 2016.

[15] Bailey AP., Hawkins LK., Missmer SA., Correia KF., Yanushpolsky EH., "Effect of body mass index on in vitro fertilization outcomes in women with polycystic ovary syndrome," Am J Obstet Gynecol., vol. 211, no. 2, pp. 163-169, 2014.

[16] Robert J Norman, Didier Dewailly, Richard S Legro TEA., "Polycystic Ovary Syndrome," Lancet, vol. 370, no. 3, pp. 685-97, 2007.

[17] Diamanti-Kandarakis E., "Role of obesity and adiposity in polycystic ovary syndrome," International Journal Obesity, vol. 31, no. 2, pp. 8-13. 2007.

[18] Huang J., Ni R., Chen X., Huang L., Mo Y., Yang D., "Metabolic abnormalities in adolescents with polycystic ovary syndrome in South China," Reprod Biol Endocrinol., vol. 8, no. 1, pp.142-149. 2010.

[19] Bassiouny YES., Rabie WA., Hassan AA., Darwish RK., "Association of the luteinizing hormone/choriogonadotropin receptor gene polymorphism with polycystic ovary syndrome," Gynecological Endocrinology, vol. 30, no. 6, pp. 428-30. 2014.

[20] Mukherjee S., Shaikh N., Khavale S., Shinde G., Meherji P., Shah N., et al., "Genetic variation in exon 17 of INSR is associated with insulin resistance and hyperandrogenemia among lean Indian women with polycystic ovary syndrome," Eur J Endocrinol., vol. 160, no. 5, pp. 855-62, 2009.

[21] Martinez-Bermejo M., Escobar-Morreale, HFE LR., "Obesity and the polycystic ovary syndrome," Minerva Endocrinol., vol. 32, no. 3, pp. 129-40, 2007.

[22] Zhang J., Hu J., Zhang C., Jiao Y., Kong X., Wang W., “Analyzed of risk factors for polycystic ovary syndrome complicated with non-alcoholic fatty liver disease," Exp Ther Med., vol. 15, no 5, pp. 4259-64, 2018.

[23] Ates S., Aydın S., Ozcan P., Soyman Z., Gokmen Karasu AF., Sevket O., "Clinical and metabolic characteristics of Turkish adolescents with polycystic ovary syndrome," J Obstet Gynaecol (Lahore)., vol. 1, no. 10, pp. 1-5, 2017.

[24] Moran L., Teede H., "Metabolic features of the reproductive phenotypes of polycystic ovary syndrome," Hum Reprod Update., vol. 15, no. 4, pp. 477-88, 2009.

[25] Saghafi-Asl M., Pirouzpanah S., Ebrahimi-Mameghani M., Asghari-Jafarabadi M., Aliashrafi S., Sadein B., "Lipid profile in relation to anthropometric indices and insulin resistance in overweight women with polycystic ovary syndrome," Heal Promot Perspect., vol. 3, no. 2, pp. 206-16, 2013.

[26] Leo V De, Musacchio MC, Cappelli V., Massaro MG, Morgante G., Petraglia F., "Genetic, hormonal and metabolic aspects of PCOS: an update," Reprod Biol Endocrinol., vol. 3, no. 4, pp. 1-17 2016.

[27] Parlak, M., Parlak, AE, \& Toslak, IE. "Etiologic Factors and Clinical Characteristics of Turkish Adolescent Girls Diagnosed with Hirsutism. Journal of Clinical and Analytical Medicine, vol. 7, no 2, pp. 205-209, 2016.

[28] Malini NA, Roy George K., "Evaluation of different ranges of LH: FSH ratios in polycystic ovarian syndrome (PCOS)-Clinical based case control study," Endocrinol Comp gene., vol. 260, no. 2, pp. 51-7, 2018.

[29] Turhan NÖ, Toppare MF, Seçkin NC, Dilmen G., "The predictive power of endocrine tests for the diagnosis of polycystic ovaries in women with oligoamenorrhea," Gynecol Obstet Invest., vol. 48, no. 3, pp. 183-6, 1999.

[30] Behbahani BM, Akbaezadeh M., "Central Obesity and BMI Associated with Different Phenotypes of Polycystic Ovary Syndrome in Adolescent," Int J of Pub Health Scie., vol. 6, no. 4, pp. 356-359, 2017. 